\title{
Detecção de metalo-beta-lactamase em Pseudomonas aeruginosa isoladas de pacientes hospitalizados em Goiânia, Estado de Goiás
}

\author{
Detection of metallo-beta-lactamase in Pseudomonas aeruginosa isolated \\ from hospitalized patients in Goiânia, State of Goiás
}

\author{
Diana Christina Pereira Santos Gonçalves ${ }^{1}$, Ana Beatriz Mori Lima ${ }^{2}$, \\ Lara Stefania Netto de Oliveira Leão ${ }^{3}$, José Rodrigues do Carmo Filho ${ }^{4}$, \\ Fabiana Cristina Pimenta ${ }^{3}$ e José Daniel Gonçalves Vieira ${ }^{3}$
}

\begin{abstract}
RESUMO
Pseudomonas aeruginosa é uma bactéria frequentemente isolada no ambiente hospitalar. Este estudo teve como objetivo avaliar o perfil de suscetibilidade de Pseudomonas aeruginosa previamente isoladas de pacientes internados em um hospital de Goiânia (Goiás-Brasil); realizar a triagem fenotípica para a produção de metalo-beta-lactamase e detectar os genes das mesmas pela técnica de "Polimerase Chain Reaction". Foram avaliadas 75 Pseudomonas aeruginosa isoladas no período de janeiro de 2005 a janeiro de 2007 . A identificação bioquímica foi realizada pelo sistema API $20 \mathrm{E}^{\circledR} \mathrm{e} 0$ antibiograma pelo método de Kirby-Bauer. Entre os 62 isolados que foram resistentes ao imipenem e à ceftazidima, 35 (56,4\%) apresentaram produção de metalo-beta-lactamase e em 26 (74,3\%) destes, foi detectado o gene bla $_{\mathrm{SPM}-1}$. A frequência de Pseudomonas aeruginosa produtoras de metalo-beta-lactamase sugere um maior controle da disseminação de resistência no ambiente hospitalar.
\end{abstract}

Palavras-chaves: Pseudomonas aeruginosa. Metalo-beta-lactamase. Infecção nosocomial. Carbapenêmicos. Multirresistência.

\begin{abstract}
Pseudomonas aeruginosa is a bacterium frequently isolated from hospital environments. This study had the aims of evaluating the susceptibility profile of Pseudomonas aeruginosa previously isolated from patients in a hospital in Goiânia (Goiás, Brazil), performing phenotypic screening for metallobeta-lactamase production and detecting its genes using the polymerase chain reaction technique. Seventy-five 75 Pseudomonas aeruginosa isolates were evaluated between January 2005 and January 2007. Biochemical identification was performed using the API $20 E^{\circledR}$ system and an antibiogram was produced using the Kirby-Bauer method. Among the 62 isolates that were resistant to imipenem and ceftazidime, 35 (56.4\%) produced metallo-betalactamase, while $26(74.3 \%)$ showed the $b l a_{\mathrm{SPM}-1}$ gene. The frequency of Pseudomonas aeruginos $a$ that produces metallo-beta-lactamase suggests that greater control over the dissemination of resistance in hospital environments is needed.
\end{abstract}

Key-words: Pseudomonas aeruginosa. Metallo-beta-lactamase. Nosocomial infection. Carbapenems. Multiresistance.

A importância clínica dos bastonetes Gram-negativos não fermentadores (BGNNF) tem aumentado significativamente devido à gravidade da infecção, elevada taxa de morbidade e mortalidade, principalmente em pacientes hospitalizados ${ }^{2}$. Esse grupo de microrganismos também apresenta resistência intrínseca a vários antimicrobianos, representando um grande desafio na terapêutica. Dentre os BGNNF, Pseudomonas aeruginosa é um dos principais agentes causadores de infecção em pacientes hospitalizados em unidades de terapia intensiva (UTI) e queimados ${ }^{15}$.

1. Programa de Pós-Graduação em Medicina Tropical e Saúde Publica do Instituto de Patologia Tropical e Saúde Pública da Universidade Federal de Goiás, Goiânia, Goiás. 2. Laboratório Rômulo Rocha, Faculdade de Farmácia, Universidade Federal de Goiás, Goiânia, Goiás. 3. Departamento de Microbiologia, Instituto de Patologia Tropical e Saúde Pública, Universidade Federal de Goiás, Goiânia, Goiás. 4. Departamento de Ciências Ambientais e Saúde, Universidade Católica de Goiás, Goiânia, Goiás.

Endereço para Correspondência: Dra. Fabiana Cristina Pimenta. Rua Delenda Rezende de Melo, esq. 1ª Avenida, Setor Universitário, 74605-050 Goiânia, G0.

e-mails: pimentaf@hotmail.com; jdgvieira62@yahoo.com.br

Tel: 5562 3209-6361

Recebido para publicação em 10/02/2009

Aceito em 20/07/2009
A utilização indiscriminada de carbapenêmicos no ambiente hospitalar promove uma pressão seletiva sobre a microbiota, o que favorece a seleção de subpopulações de microrganismos com sensibilidade diminuída ou resistente a esses antimicrobianos ${ }^{6}$.

O isolamento de Pseudomonas aeruginosa multirresistentes tem sido frequente nos últimos anos. Vários agentes antimicrobianos têm se tornado menos ativos, reduzindo o número de opções terapêuticas e aumentando o impacto clínico de infecções nosocomiais. Dentre as preocupações relacionadas à resistência antimicrobiana, destacam-se a produção de metalo-betalactamase $^{12}$.

Pseudomonas aeruginosa produtora de metalo-betalactamase (MBL) tem sido reportada como importante causa de infecções hospitalares. Apesar da prevalência destes microrganismos em hospitais ainda ser pouco investigada, os mesmos estão associados aos casos de disseminação clonal e surtos hospitalares ${ }^{5}$.

A emergência de bactérias produtoras de MBL requer mudanças na rotina dos laboratórios de Microbiologia, 
adequando métodos capazes de detectar a sua produção. Entretanto, representa um desafio, pois não existe um consenso sobre a padronização de metodologias para a detecção da produção dessas enzimas ${ }^{13}$.

0 presente estudo teve como objetivo avaliar o perfil de suscetibilidade de Pseudomonas aeruginosa previamente isoladas de pacientes internados em um hospital de Goiânia (GO); realizar a triagem fenotípica para a produção de MBL dos isolados que se apresentarem resistentes ao imipenem e à ceftazidima e detectar os genes das MBL $\left(b l a_{\mathrm{IMP}-1}, b l a_{\mathrm{IMP}-2}, b l a_{\mathrm{VIM}-1}\right.$, $b l a_{\mathrm{VIM}-2}$ e $\left.b l a_{\mathrm{SPM}-1}\right)$.

\section{MATERIAL E MÉTODOS}

0 estudo foi desenvolvido em um hospital da rede privada localizado no município de Goiânia (G0) em parceria com 0 Instituto de Patologia Tropical e Saúde Pública da Universidade Federal de Goiás. O hospital possui um total de 64 leitos, destes 11 são destinados à Unidade de Terapia Intensiva. Foram avaliadas 75 Pseudomonas aeruginosa isoladas de pacientes internados, oriundas de diversos espécimes clínicos.

0 critério de inclusão dos pacientes no estudo foi a admissão nas unidades clínicas do referido hospital, no período de janeiro de 2005 a janeiro de 2007, independente de gênero e doença de base. 0 protocolo de investigação $\left(n^{0} 236 / 07\right)$ foi aprovado pelo Comitê de Ética Regional do Hospital Geral de Goiânia atendendo à resolução 196/96.

Os meios de cultura utilizados foram escolhidos de acordo com os espécimes clínicos analisados ${ }^{9}$. Amostras de urina foram semeadas em ágar Cled (Biobrás) e ágar MacConkey (Difco). Amostras de secreções diversas foram semeadas em ágar base (Biobrás) com 5\% de sangue de carneiro, ágar chocolate e caldo tioglicolato (Difco). Amostras provenientes de hemoculturas foram inoculadas em meio Hemobac trifásico (Probac).

A identificação dos isolados foi feita pelo aspecto morfológico das colônias, odor, produção de pigmentos, características morfotintoriais e teste da oxidase ${ }^{9}$. Para a identificação bioquímica foi utilizado o sistema API 20E ${ }^{\circledR}$ (bioMérieux).

0 perfil de suscetibilidade dos isolados foi realizado pelo teste de difusão em ágar e os seguintes antimicrobianos foram avaliados: amicacina $(30 \mu \mathrm{g})$; aztreonam $(30 \mu \mathrm{g})$; cefepima $(30 \mu \mathrm{g})$; ceftazidima $(30 \mu \mathrm{g})$; ciprofloxacina $(5 \mu \mathrm{g})$; gentamicina (10 $\mu \mathrm{g})$; imipenem $(10 \mu \mathrm{g})$; piperacilina-tazobactam $(75 / 10 \mu \mathrm{g}) \mathrm{e}$ tobramicina $(30 \mu \mathrm{g})$. Os resultados foram reportados como sensível, resistência intermediária ou resistente conforme recomendações da CLSI (Clinical and Laboratory Standards Institute) ${ }^{4}$.

Pseudomonas aeruginosa com resistência ao imipenem e ceftazidima foram submetidas à avaliação da produção de MBL pelo método do disco aproximação como descrito por Arakawa cols $^{1}$. Foi utilizado o ácido 2-mercaptopropiônico (2MPA) e o ácido etilenodiaminotetracético (EDTA). Os substratos

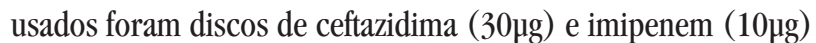
posicionados ao lado de um disco de papel de filtro contendo a solução do agente quelante.
A presença de uma zona de expansão ou distorção no halo de inibição do crescimento entre os discos contendo o 2MPA ou EDTA e os discos de ceftazidima e imipenem indica que o isolado é produtor de uma metalo-beta-lactamase ${ }^{1}$.

Pseudomonas aeruginosa classificadas fenotipicamente como produtoras de MBL pelo teste de aproximação de discos foram avaliadas pela técnica da reação de polimerase em cadeia (PCR) para identificar a presença de genes que codificam a produção de MBL.

Os oligonucleotídeos inicializadores utilizados foram: $b l a_{\text {IMP-1 }} \mathrm{F}(5$ ' CTACCGCAGCAGAGTCTTTGC3') e R(5'GAACAACCAGTTTTGCCTTACC3'). A reação de amplificação do gene $b l a_{\mathrm{IMP}-1}$ produziu um produto com 587 pares de base. Para reação de multiplex, os oligonucleotídeos iniciadores utilizados para $b l a_{\mathrm{IMP}-2,} b l a_{\mathrm{VIM}-1}$ e $b l a_{\mathrm{VIM}-2}$ foram: F(5'GGCAGTCGCCCTAAAACAAA3') e R (5'TAGTTACTTGGCTGTGATGG3'), F(5'TCTACATGACCGCGTCTGTC3') e R (5'TGTGCTTTGACAACGTTCGC3'), F(5ATGTTCAAACTTTTGAGTAGTAAG3') e R(5'CTACTCAACGACTGAGCG $\left.3^{\prime}\right)^{1419}$. A reação de amplificação dos genes $b l a_{\mathrm{IMP}-2,} b l a_{\mathrm{VIM}-1}$ e $b l a_{\mathrm{VIM}-2}$ originaram respectivamente produtos de: 737 pares de base, 920 pares de base e 801 pares de base.

A amplificação do gene bla $a_{\mathrm{SPM}-1}$ utilizou o iniciador $\mathrm{F}(5$ ' CCTACAATCTAACGGCGACC3') e R(5'TCGCCGTGTCCAGGTATAAC3') ${ }^{17}$. A reação de amplificação do gene $b l a_{\mathrm{SPM}-1}$ resultou em um produto com 650 pares de base.

\section{RESULTADOS}

Foram avaliados 75 Pseudomonas aeruginosa sendo que $29(38,7 \%)$ foram isoladas de amostras de urina, 21 (28\%) de lavado broncoalveolar, 8 (10,7\%) de secreção abdominal, 7 (9,3\%) debridamento de escara, 5 (6,7\%) de ferida operatória, 3 (4\%) de hemoculturas, 1 (1,3\%) de escarro e 1 (1,3\%) ponta de catéter. A distribuição dos 75 isolados de acordo com os setores de atendimento dos pacientes no hospital avaliado está apresentada na Tabela 1. A maioria $(62,7 \%)$ das amostras foi isolada de pacientes internados na UTI.

Os isolados avaliados apresentaram perfil de resistência a diferentes classes de antimicrobianos. A maior taxa de resistência foi observada frente à ciprofloxacina (97,3\%, 73 isolados), seguida pela ceftazidima $(90,7 \%, 68$ isolados) e pela cefepima (88\%, 66 isolados). A menor taxa de resistência foi observada frente ao aztreonam (30,7\%, 23 isolados) (Tabela 2).

TABELA 1

Distribuição das Pseudomonas aeruginosa por setores de um hospital de Goiânia, GO, onde foram isoladas 75 amostras no período de janeiro de 2005 a janeiro de 2007.

\begin{tabular}{lrr}
\hline Setor do hospital & Número & Porcentagem \\
\hline Unidade de Terapia Itensiva & 47 & 62,7 \\
Apartamento & 13 & 17,3 \\
Enfermaria & 11 & 14,6 \\
Ambulatório & 4 & 5,4 \\
\hline Total & 75 & 100,0 \\
\hline
\end{tabular}


Entre as Pseudomonas aeruginosa avaliadas 62 isolados foram resistentes ao imipenem e cefetazidima. Destes 35 (56,4\%) foram fenotipicamente produtoras de metalo-beta-lactamases (MBL) (Tabela 3). 0 2-mercaptopropiônico foi o inibidor que apresentou melhor atividade em relação ao EDTA na detecção dos isolados produtores (Tabela 3).

\section{TABELA 2}

Perfil de suscetibilidade de 75 amostras de Pseudomonas aeruginosa isoladas de diversos espécimes clínicos de pacientes internados em um hospital de Goiânia, GO.

\begin{tabular}{|c|c|c|c|c|c|c|c|c|}
\hline \multirow[b]{2}{*}{ Antimicrobiano } & \multicolumn{2}{|c|}{ Resistente } & \multicolumn{2}{|c|}{ Intermediário } & \multicolumn{2}{|c|}{ Sensível } & \multicolumn{2}{|c|}{ Não realizado } \\
\hline & $\overline{\mathrm{n}^{\mathrm{o}}}$ & $\overline{\%}$ & $\mathrm{n}^{0}$ & $\%$ & $\overline{\mathrm{n}^{0}}$ & $\overline{\%}$ & $\mathrm{n}^{0}$ & $\%$ \\
\hline Imipenem & 62 & 82,7 & 13 & 17,3 & - & - & - & - \\
\hline Ceftazidima & 68 & 90,7 & - & - & 7 & 9,3 & - & - \\
\hline Aztreonam & 23 & 30,7 & 15 & 20,0 & 37 & 49,3 & - & - \\
\hline Ciprofloxacina & 73 & 97,3 & - & - & 2 & 2,7 & - & - \\
\hline Piperac/Tazobac & 36 & 48,0 & 4 & 5,3 & 13 & 17,3 & 22 & 29,4 \\
\hline Amicacina & 59 & 78,7 & 2 & 2,7 & 14 & 8,6 & - & - \\
\hline Gentamicina & 63 & 84,0 & 2 & 2,7 & 9 & 12 & 1 & 1,3 \\
\hline Tobramicina & 58 & 77,4 & 3 & 4,0 & 7 & 9,3 & 7 & 9,3 \\
\hline Cefepima & 66 & 88,0 & 5 & 6,6 & 4 & 5,4 & - & - \\
\hline
\end{tabular}

\section{TABELA 3}

Detecção de produção de metalo-beta-lactamase utilizando como inibidor EDTA/2MPA e o substrato ceftazidima de 62 amostras de Pseudomonas aeruginosa isoladas de pacientes internados em um hospital em Goiânia, GO.

\begin{tabular}{lcc}
\hline MBL & 2MPA/CAZ & EDTA/CAZ \\
\hline Teste positivo & 35 & 6 \\
Teste negativo & 27 & 56 \\
\hline Total & $\mathbf{6 2}$ & $\mathbf{6 2}$ \\
\hline
\end{tabular}

MBL: metalo-beta-lactamase, 2MPA/CAZ: ácido 2-mercaptopropiônico/ceftazidima, EDTA: etileno diamino tetraacético.

0 gene $b l a_{\text {SPM-1 }}$ foi detectado em $26(74,3 \%)$ dos 35 isolados de Pseudomonas aeruginosa que apresentaram fenótipo positivo. Os genes $b l a_{\mathrm{IMP}-1}, b l a_{\mathrm{IMP}-2}, b l a_{\mathrm{VIM}-1}$ e $b l a_{\mathrm{VIM}-2}$ não foram detectados.

\section{DISCUSSÃO}

A produção de MBL foi verificada, pelo método fenotípico de disco aproximação, em 35/62 (56,4\%) das Pseudomonas aeruginosa analisadas. 0 teste de disco aproximação, empregado para detectar MBL, pode apresentar boa sensibilidade e especificidade. Porém, esses resultados podem variar de acordo com a espécie bacteriana testada, o substrato e o agente quelante utilizado.

Segundo Arakawa cols ${ }^{1}$, o agente quelante 2MPA apresentou melhor atividade (100\%) quando comparado ao EDTA utilizado na detecção de amostras produtoras de MBL utilizando-se a ceftazidima como substrato. Neste estudo, o teste com 2MPA permitiu verificar a produção de MBL em 35 isolados, enquanto utilizando EDTA, a produção foi verificada em 6.

Em outro estudo realizado, o 2MPA apresentou $100 \%$ de atividade na detecção de amostras de Acinetobacter spp produtoras de MBL; porém, falhou em detectar 10,5\% das
Pseudomonas aeruginosa analisadas. Em contrapartida, o EDTA apresentou melhor detecção da produção de MBL em amostras de Pseudomonas aeruginosa (100\%), mas falhou em detectar $6 \%$ das amostras de Acinetobacter $s p p^{10}$.

A taxa de Pseudomonas aeruginosa produtora de MBL encontrada nesse estudo está em concordância com os resultados descritos por Jayakumar cols ${ }^{8}$, que verificaram $46,7 \%$ de produção de MBL em Pseudomonas aeruginosa com resistência a imipenem e meropenem e isoladas de pacientes de um hospital de cuidados terciários.

A maioria das Pseudomonas aeruginosa avaliadas nesse estudo foi isolada de amostras de urina $(38,7 \%)$, seguido pelo lavado broncoalveolar com $28 \%$ e secreção abdominal (10,7\%). Villas Bôas cols ${ }^{18}$ descreveram uma frequência de $26,4 \%$, de infecções do trato urinário, em idosos internados em um hospital universitário.

0 perfil de resistência, dos isolados avaliados, ao imipenem e a ceftazidima foi de 82,7 e 90,7\%, respectivamente, enquanto Santos Filho cols ${ }^{16}$ avaliaram 198 Pseudomonas aeruginosa isoladas de diversos espécimes clínicos, hospitalares e comunitárias relataram um percentual de resistência de 19,7\% ao imipenem e de $15,2 \%$ à ceftazidima, com 10,1\% de resistência cruzada aos dois antimicrobianos. Entre esses isolados, apenas 2\% produziram MBL, dados inferiores aos detectados nesse estudo.

0 gene $b l a_{\text {SPM-1 }}$ foi detectado em 26 (74,3\%) isolados. Entretanto, em 9 (25,7\%) isolados com fenótipo positivo para MBL, não foram detectados nenhum dos genes avaliados. Estudos realizados no Brasil demonstram que Pseudomonas aeruginosa portadora do gene $b l a_{\mathrm{SPM}-1}$ está disseminada pelo país e a taxa de isolados produtores de MBL é variável em diferentes regiões ${ }^{31620}$. As infecções causadas por este agente produtor da carbapenemase SPM-1 está relacionada com elevada mortalidade, como demonstrado no estudo que avaliou a produção da MBL em 76 Pseudomonas aeruginosa isoladas de pacientes com infecção da corrente sanguínea, sendo que 23 (30,3\%) apresentaram fenótipo MBL e a taxa de mortalidade foi de $85,7 \%$ entre os pacientes infectados por Pseudomonas aeruginosa produtora de $M B L^{11}$.

A detecção de genes de resistência em isolados clínicos e a avaliação de seu perfil de suscetibilidade fornecem dados importantes para a racionalização da terapia antimicrobiana e redução da taxa de mortalidade. Porém, devido ao impacto da produção de MBL no contexto hospitalar, estudos adicionais são necessários para a elaboração e implementação de medidas mais efetivas de prevenção e controle das infecções nosocomiais, diminuindo custos hospitalares e qualificando os serviços oferecidos pelas equipes de saúde.

Concluindo, os dados apresentados neste estudo mostraram taxas elevadas de Pseudomonas aeruginosa produtoras de MBL, dificultando assim as opções para tratamentos e a necessidade de vigilância individualizada do perfil de resistência em instituições de saúde. Essas informações devem auxiliar na adoção de medidas efetivas para o uso racional dos antimicrobianos e redução da disseminação de microrganismos resistentes nas instituições de saúde. 


\section{AGRADECIMENTOS}

Os autores agradecem ao Programa de Pós-graduação em Medicina Tropical do Instituto de Patologia Tropical e Saúde Pública da Universidade Federal de Goiás e a Fundação de Amparo a Pesquisa da Universidade Federal de Goiás (FUNAPE/UFG) pelo apoio financeiro.

\section{REFERÊNCIAS}

1. Arakawa Y, Shibata N, Shibayama K, Kurokawa H, Yagi T, Fujiwara H, Goto M. Convenient test for screening metallo- $\beta$-Lactamase-producing gram-negative bacteria by using thiol compounds. Journal Clinical Microbiology 38: 40-43, 2000

2. Bradley JS, Garau J, Lode H, Rolston KVI, Wilson SE, Quinn JP. Carbapenems in clinical practice: a guide to their use in serious infection. International Journal Antimicrobial Agents 11: 93-100, 1999.

3. Castanheira M, Fritsche TR, Sader HS, Jones RN, Doi Y, Garcia DO, Paterson DL RmtD 16S RNA methylase in Epidemiologically Unrelated SPM-1-Producing Pseudomonas aeruginosa Isolates from Brazil. Antimicrobial Agents and Chemoterapy 52: 1587-1588, 2008.

4. Clinical and Laboratory Standards Institute. Performance Standards for Antimicrobial Susceptibility Testing. Sixteenth information supplement. CSLI document M 100-S16. Wayne, PA, USA, 2006.

5. Crespo MP, Woodford N, Sinclair A, Kaufmann ME, Turton J, Glover J, Velez JD, Castaneda CR, Recable M, Livemore DM. Outbreak of carbapenem-resistant Pseudomonas aeruginosa producing VIM-8, a novel metallo- $\beta$-lactamase, in a tertiary care center in Cali, Colombia. Journal Clinical Microbiology 42: 50945101, 2004

6. Gales AC, Menezes LC, Silbert S, Sader HS. Dissemination in distinct Brazilian regions of an epidemic carbapenêmicos-resistant Pseudomonas aeruginosa producing SPM metallo- $\beta$-lactamase. Journal Antimicrobial Chemotherapy 52: 699-702, 2003

7. Hirakata Y, Yamaguchi T, Nakano M, Izumikawa K, Mine M, Aoki S, Kondoh A, Matsuda J, Hirayawa M. Ynagihara K, Miyazaki Y, Tomono K, Yamada Y Kamihira S, Kohno S. Clinical and bacteriological characteristics of IMP-type metallo- $\beta$ lactamese-producing Pseudomonas aeruginosa. Clinical Infectious Diseases 37: 26-32, 2003.

8. Javakumar S, Appalaraju B. Prevalence of multi and pan drug resistant Pseudomonas aeruginosa with respect to ESBL and MBL in a terciary care hospital. Indian Journal Pathology Microbiology 50: 922-925, 2007.
9. Koneman EW, Win Jr JWC, Allen SD, Janda WM, Gary WP, Schreckenberg PC, Woods GL, Lippincott W. The Nonfermentative Gram-Negative Bacilli. In: Koneman EW, Win Jr JWC, Allen SD, Janda WM, Gary WP, Schreckenberg PC, Woods GL, Lippincott W (ed) Koneman's Color Atlas and Textbook of Diagnostic Microbiology, Sixth Edition, Lippincott Willians \& Wilkins, Baltimore, Chapter 7, p. 303-391, 2006.

10. Lee K, Lee WG, Uh Y, Ha GY, Cho J, Chong Y. VIM- and IMP-type metallo- $\beta$ lactamase-producing Pseudomonas spp. and Acinetobacter spp. in Korean hospitals. Emerging Infectious Diseases 9: 868-871, 2003.

11. Marra AR, Pereira CAP, Gales AC, Menezes LC, Cal RGR, Souza JMA, Edmond MB, Faro C, Wey SB. Bloodstream Infections with Metallo- $\beta$-Lactamase-Producing Pseudomonas aeruginosa: Epidemiology, Microbiology and Clinical Outcomes. Antimicrobial Agents Chemotherapy 50: 388-390, 2006

12. Pfaller MA, Jones RN, Doern GV, Kugler K. Bacterial pathogens isolated from patients with bloodstream infection: frequencies of occurrence and antimicrobial susceptibility patterns from the SENTRY antimicrobial surveillance program (United States and Canada, 1997). Antimicrobial Agents Chemotherapy 42:17621770, 1998.

13. Picão RC, Andrade S, Nicoletti AG, Campana EH, Moraes GC, Mendes RE, Gales AC. Metallo- $\beta$-lactamase detection: comparative evoluation of double-disk synergy versus combined disk tests for IMP, GIM, SIM or VIM-producing isolates. Journal Clinical Microbiology 46: 2028-2047, 2008.

14. Poirel L, Naas T, Nicolas D, Collet L, Bellais S, Cavallo JD, Nordmann P. Characterization of VIM-2, a carbapenêmicos-hydrolyzing metallo- $\beta$-lactamase and its plasmid- and integron-borne gene from a Pseudomonas aeruginos $a$ clinical isolate in France. Antimicrobial Agents Chemotherapy 44: 891-897, 2000.

15. Saiman L, Siegel J. Infection control in cystic fibrosis. Clinical Microbiology Review 17: 57-71, 2004.

16. Santos Filho L, Santos IB, Assis AML, Xavier DE. Determinação da produção de metalo $\beta$-lactamases em amostras de Pseudomomas aeruginosa isoladas em João Pessoa, Paraíba. Jornal Brasileiro de Patologia e Medicina Laboratorial 38: 79-84, 2002

17. Toleman MA, Simm AM, Murphy TA, Gales AC, Biedenbach DJ, Jones RN, Walsh TR. Molecular characterization of SPM-1, a novel metallo- $\beta$-lactamase isolated in Latin America: report from the SENTRY Antimicrobial Surveillance Program. Journal Antimicrobial Chemotherapy 50: 673-679, 2002.

18. Villas Bôas PJF, Ruiz T. Ocorrência de infecção hospitalar em idosos internados em hospital universitário. Revista de Saúde Pública 38: 372-378, 2004.

19. Yan JJ, Ko WC, Wu JJ. Identification of a plasmid encoding SHV-12, TEM-1 and a variant of IMP-2 metallo- $\beta$-lactamase, IMP-8, from a clinical isolate of Klebsiella pneumoniae. Antimicrobial Agents Chemotherapy 45: 2368-2371, 2001.

20. Zavascki AP, Gaspareto PB, Martins AF, Gonçalves AL, Barth AL. Outbreak of carbapenem-resistant Pseudomonas aeruginosa producing SPM-1 metallo$\beta$-lactamase in a teaching hospital in southern Brazil. Journal of Antimicrobial Chemotherapy 56: 1148-1151, 2005 